\title{
Semi-invariant lightlike submanifolds of golden semi-Riemannian manifolds
}

\author{
Nergiz (ÖNEN) POYRAZ ${ }^{1, *}$, Burçin DOĞAN² \\ ${ }^{1}$ Çukurova University, Faculty of Arts and Sciences, Department of Mathematics, Adana. \\ ${ }^{2}$ Malatya Turgut Özal University, Faculty of Engineering and Naturel Sciences, \\ Department of Basic Sciences of Engineering, Malatya. \\ Geliş Tarihi (Received Date): 08.03.2019 \\ Kabul Tarihi (Accepted Date): 25.07.2019
}

\begin{abstract}
In this study, we introduce semi-invariant lightlike submanifolds of golden semiRiemannian manifolds. We find some conditions for integrability of distributions. Morever, we investigate totally geodesic and mixed geodesic distributions of such a submanifold. The paper also contains an example.
\end{abstract}

Keywords: Lightlike submanifold, Golden semi-Riemann manifold, semi-invariant submanifold.

\section{Altın semi-Riemann manifoldların semi-invaryant lightlike altmanifoldları}

\section{$\ddot{\mathbf{O} z}$}

$\mathrm{Bu}$ makalede altın semi-Riemann manifoldlartn semi-invaryant lightlike altmanifoldlarını çalıştık. Distribüsyonların integrallenebilirliği için bazı koşullar bulduk. Ayrlca, böyle bir altmanifoldun tamamen geodezik ve mixed geodezik distribüsyonlarını inceledik. Makale, bir adet örnek içermektedir.

Anahtar kelimeler: Lightlike altmanifold, Altın semi-Riemann manifold, semi-invaryant altmanifold.

\footnotetext{
*Nergiz (ÖNEN) POYRAZ, nonen@cu.edu.tr, https://orcid.org/0000-0002-8110-712X

Burçin DOĞAN, burcin.dogan@ ozal.edu.tr, https://orcid.org/0000-0001-8386-213X
} 


\section{Introduction}

The applications in general relativity of degenerate submanifolds of semi-Riemannian manifolds have made such submanifolds one of the most important fields of study in differential geometry. In contrast to the Riemannian and semi-Riemannian submanifolds, the intersection of the normal bundle and the tangent bundle in lightlike submanifolds is not zero. Thus, studying the geometry of lightlike submanifolds is more difficult and complicated. The first studies on lightlike submanifolds are belong to Duggal-Bejancu [7] and Kupeli [17]. Later, Duggal and Şahin introduced a book on lightlike geometry [10]. Many authors have studied on lightlike submanifolds in various spaces, for example $[2,3,4,5,8,9,16]$.

The geometries of some manifolds with differentiable geometric structures, which are quite suitable tools for the differential geometry, are interesting. These manifolds and the transformations between them have been studied extensively in differential geometry. One of these structures is $f$-structure which is described by Yano in [20]. A tensor field $\phi$ on manifold $\breve{N}$ is an $f$-structure if it satisfies that $\phi^{3}+\phi=0$. The almost complex and contact structures are obvious examples of $f$-structures. Goldberg and Yano introduced polynomial structures of degree $d$ in [14] by generalizing $f$-structures. A polynomial structure satisfies the equation $\theta(f)=f_{d}+a_{d} f_{d-1}+\ldots+a_{2} f+a_{1} I=0$, where $a_{1}, a_{2}, \ldots, a_{d}$ are real numbers and $I$ is the identity tensor of type $(1,1)$.

Golden proportion $\varphi$ is the real positive root of the equation $x^{2}-x-1=0$ (thus, $\left.\varphi=\frac{1+\sqrt{5}}{2} \approx 1.618 \ldots\right)$. Inspired by the Golden proportion, Crasmareanu and Hretcanu defined $\breve{P}$ golden structure which is a tensor field satisfying $\breve{P}^{2}-\breve{P}-I=0$ on $\breve{N}$ [6]. In the same paper, it is shown that $\breve{P}$ is an automorphism of $T \breve{N}$ and its eigenvalues are $\varphi$ and $1-\varphi$. We note that for golden structures, $\breve{P} \neq \varphi I$. If $\breve{P}=\varphi I$, then its minimal polynomial is $X-\varphi$. But, the minimal polynomial of the golden structure $\breve{P}$ is $X^{2}-X-1$ and it is a contraction.

A Riemannian manifold $\breve{N}$ with a $\breve{P}$ golden structure is named as a golden Riemannian manifold in $[6,15]$ and invariant submanifolds of such manifolds were studied in [15]. Later, Erdoğan and Yıldırım researched totally umbilical semi-invariant manifolds of golden manifold in [12]. Many authors investigated the properties of golden structures and golden manifolds in Riemannian [13, 18] and semi-Riemannian geometry. Submanifolds of golden manifolds in degenerate geometry were firstly studied by Poyraz and Yaşar in [19]. In the study, they introduced lightlike hypersurfaces of a golden semi-Riemannian manifold and investigated geometry of such hypersurfaces in detail. Acet studied lightlike hypersurfaces of metallic semi-Riemannian manifolds in [1] and Erdoğan researched transversal lightlike submanifolds of metallic semiRiemannian manifolds in [11].

In this study, we introduce semi-invariant lightlike submanifolds of golden semiRiemannian manifolds. We find some conditions for integrability of distributions. Morever, we investigate totally geodesic and mixed geodesic distributions of such a submanifold. The paper also contains an example. 


\section{Preliminaries}

A golden structure $\breve{P}$ on a differentiable manifold $\breve{N}$ is defined as

$\breve{P}^{2}=\breve{P}+I$

and if

$\breve{g}(\breve{P} X, Y)=\breve{g}(X, \breve{P} Y)$

then $(\breve{N}, \breve{g}, \breve{P})$ is called a golden semi-Riemannian manifold [18].

Let $(\breve{N}, \breve{g}, \breve{P})$ be a semi-Riemannian golden manifold. Then for any $X, Y \in \Gamma(T \breve{N})$, equation (2.2) can be written as

$\breve{g}(\breve{P} X, \breve{P} Y)=\breve{g}(\breve{P} X, Y)+\breve{g}(X, Y)$.

We denote real space forms with constant sectional curvatures $c_{p}$ and $c_{q}$, by $N_{p}^{\prime}$ and $N_{q}^{\prime}$, respectively. Then similar calculations of semi-Riemannian product real space form (see [21]), the Riemannian curvature tensor $\bar{R}$ of a locally Golden product space form $\left(\breve{N}=N_{p}^{\prime}\left(c_{p}\right) \times N_{q}^{\prime}\left(c_{q}\right), \breve{g}, \breve{P}\right)$ is calculated as the following

$$
\bar{R}(X, Y) Z=\begin{gathered}
\left(-\frac{(1-\varphi) c_{p}-\varphi c_{q}}{2 \sqrt{5}}\right)\{\breve{g}(Y, Z) X-\breve{g}(X, Z) Y+\breve{g}(\breve{P} Y, Z) \breve{P} X-\breve{g}(\breve{P} X, Z) \breve{P} Y\} \\
+\left(-\frac{(1-\varphi) c_{p}+\varphi c_{q}}{4}\right)\{\breve{g}(\breve{P} Y, Z) X-\breve{g}(\breve{P} X, Z) Y+\breve{g}(Y, Z) \breve{P} X-\breve{g}(X, Z) \breve{P} Y\} .
\end{gathered}
$$

Let $(\breve{N}, \breve{g})$ be a real $(m+n)$-dimensional semi-Riemannian manifold with index $q$, such that $m, n \geq 1,1 \leq q \leq m+n+1$ and $\left(N^{\prime}, g\right)$ be a $m$-dimensional submanifold of $\bar{N}$, where $g$ is the induced metric of $\breve{g}$ on $N^{\prime}$. If $\breve{g}$ is degenerate on the tangent bundle $T N^{\prime}$ of $N^{\prime}$ the $N^{\prime}$ is named a lightlike submanifold of $\breve{N}$. For a degenerate metric $g$ on $N^{\prime}$

$$
T N^{\prime \perp}=\cup\left\{u \in T_{x} \vec{N}: \breve{g}(u, v)=0, \forall v \in T_{x} N^{\prime}, x \in N^{\prime}\right\}
$$

is a degenerate $n$-dimensional subspace of $T_{x} \breve{N}$. Since, both $T_{x} N^{\prime}$ and $T_{x} N^{\prime \perp}$ are degenerate orthonormal distributions, there exists a radical (null) space $\operatorname{Rad}\left(T_{x} N^{\prime}\right)$ such that $\operatorname{Rad}\left(T_{x} N^{\prime}\right)=T_{x} N^{\prime} \cap T_{x} N^{\prime \perp}$. If $\operatorname{rank}\left(\operatorname{Rad}\left(T_{x} N^{\prime}\right)\right)=r>0$, then $N^{\prime}$ is an $r$-lightlike submanifold of $\vec{N}$.

Denote the complementary distributions of $\operatorname{Rad}\left(T N^{\prime}\right)$ in $T N^{\prime}$ and $T N^{\prime \perp}$ by $S\left(T N^{\prime}\right)$ and $S\left(T N^{\prime}\right)$, respectively. Also, let $\operatorname{tr}\left(T N^{\prime}\right)$ and $\operatorname{ltr}\left(T N^{\prime}\right)$ be complementary (but not orthogonal) vector bundles to $T N^{\prime}$ in $T \bar{N}_{\mathrm{I}^{\prime}}$, and $\operatorname{Rad}\left(T N^{\prime}\right)$ in $S\left(T N^{\prime}\right)^{\perp}$. Thus, we can write 


$$
\begin{aligned}
& T N^{\prime}=S\left(T N^{\prime}\right) \perp \operatorname{Rad}\left(T N^{\prime}\right) \\
& \operatorname{tr}\left(T N^{\prime}\right)=\operatorname{ltr}\left(T N^{\prime}\right) \perp S\left(T N^{\prime}\right), \\
& T N_{N^{\prime}}=T N^{\prime} \oplus \operatorname{tr}\left(T N^{\prime}\right)=\left(\operatorname{Rad}\left(T N^{\prime}\right) \oplus \operatorname{ltr}\left(T N^{\prime}\right)\right) \perp S\left(T N^{\prime}\right) \perp S\left(T N^{\prime}\right),
\end{aligned}
$$

where $S\left(T N^{\prime}\right), S\left(T N^{\prime}\right), \operatorname{tr}\left(T N^{\prime}\right)$ and $\operatorname{ltr}\left(T N^{\prime}\right)$ are called as screen bundle, screen transversal bundle, transversal bundle and lightlike transversal bundle, respectively.

Theorem 2.1. Let $\left(N^{\prime}, g, S\left(T N^{\prime}\right), S\left(T N^{\prime \perp}\right)\right)$ be an $r$-lightlike submanifold of a semiRiemannian manifold $(\breve{N}, \breve{g})$. Assume that $U$ is a coordinate neighbourhood of $N^{\prime}$ and $E_{i}, i \in\{1, \ldots, r\}$ is a basis of $\Gamma\left(\operatorname{Rad}\left(T N^{\prime}\right)\right)_{\mid}$. Then, there exist a complementary vector subbundle $\operatorname{ltr}\left(T N^{\prime}\right)$ of $\operatorname{Rad}\left(T N^{\prime}\right)$ in $S\left(T N^{\prime \perp}\right)^{\perp}$ and a basis $\left\{N_{i}\right\}, i \in\{1, . ., r\}$ of $\Gamma\left(\operatorname{ltr}\left(T N^{\prime}\right)\right)_{\left.\right|_{v}}$ such that

$\breve{g}\left(N_{i}, E_{j}\right)=\delta_{i j}, \quad \breve{g}\left(N_{i}, N_{j}\right)=0$,

for any $i, j \in\{1, \ldots, r\}$.

We say that a submanifold $\left(N^{\prime}, g, S\left(T N^{\prime}\right), S\left(T N^{\prime \perp}\right)\right)$ of $\breve{N}$ is

Case 1: $r$-lightlike if $r<\min \{m, n\}$,

Case 2: Coisotropic if $r=n<m, S\left(T N^{\prime \perp}\right)=\{0\}$,

Case 3: Isotropic if $r=m<n, S\left(T N^{\prime}\right)=\{0\}$,

Case 4: Totally lightlike if $r=m=n, S\left(T N^{\prime}\right)=\{0\}=S\left(T N^{\prime \perp}\right)$.

Let $\bar{\nabla}$ be the Levi-Civita connection on $\bar{N}$. The Gauss and Weingarten formulas are given by

$$
\begin{aligned}
& \bar{\nabla}_{X} Y=\nabla_{X} Y+h(X, Y), \\
& \bar{\nabla}_{X} U=-A_{U} X+\nabla_{X}^{t} U,
\end{aligned}
$$

for any $X, Y \in \Gamma\left(T N^{\prime}\right)$ and $U \in \operatorname{tr}\left(T N^{\prime}\right)$, where $\left\{\nabla_{X} Y, A_{V} X\right\}$ and $\left\{h(X, Y), \nabla_{X}^{t} U\right\}$ belong to $\Gamma\left(T N^{\prime}\right)$ and $\Gamma\left(\operatorname{tr}\left(T N^{\prime}\right)\right)$, respectively. $\nabla$ and $\nabla^{t}$ are linear connections on $N^{\prime}$ and on the vector bundle $\operatorname{tr}\left(T N^{\prime}\right)$, respectively. Using (2.8), considering the projection morphisms $L$ and $S$ of $\operatorname{tr}\left(T N^{\prime}\right)$ on $\operatorname{tr}\left(T N^{\prime}\right)$ and $S\left(T N^{\perp}\right)$, respectively, from (2.10) and (2.11), we get

$$
\begin{aligned}
& \bar{\nabla}_{X} Y=\nabla_{X} Y+h^{l}(X, Y)+h^{s}(X, Y), \\
& \bar{\nabla}_{X} N=-A_{N} X+\nabla_{X}^{l} N+D^{s}(X, N), \\
& \bar{\nabla}_{X} L=-A_{L} X+\nabla_{X}^{s} L+D^{l}(X, L),
\end{aligned}
$$

for any $X, Y \in \Gamma\left(T N^{\prime}\right), \quad N \in \Gamma\left(\operatorname{ltr}\left(T N^{\prime}\right)\right)$ and $L \in \Gamma\left(S\left(T N^{\prime \perp}\right)\right)$, where $h^{l}(X, Y)=\operatorname{Lh}(X, Y)$, $h^{s}(X, Y)=S h(X, Y), \quad \nabla_{X}^{l} N, D^{l}(X, L) \in \Gamma\left(\operatorname{ltr}\left(T N^{\prime}\right)\right), \quad \nabla_{X}^{s} L, D^{s}(X, N) \in \Gamma\left(S\left(T N^{\prime \perp}\right)\right) \quad$ and $\nabla_{X} Y, A_{N} X, A_{L} X \in \Gamma\left(T N^{\prime}\right)$ [8]. Then, using (2.12)-(2.14) and $\bar{\nabla}$ metric connection, we derive 
$\breve{g}\left(h^{s}(X, Y), L\right)+\breve{g}\left(Y, D^{l}(X, L)\right)=g\left(A_{L} X, Y\right)$,

$\breve{g}\left(D^{s}(X, N), L\right)=\breve{g}\left(A_{L} X, N\right)$.

Let $J$ be a projection of $T N^{\prime}$ on $S\left(T N^{\prime}\right)$. From (2.6) we derive

$\nabla_{X} J Y=\nabla_{X}^{*} J Y+h^{*}(X, J Y)$,

$\nabla_{X} E=-A_{E}^{*} X+\nabla_{X}^{* t} E$,

for any $X, Y \in \Gamma\left(T N^{\prime}\right)$ and $E \in \Gamma\left(\operatorname{Rad}\left(T N^{\prime}\right)\right)$, where $\left\{\nabla_{X}^{*} J Y, A_{E}^{*} X\right\}$ and $\left\{h^{*}(X, J Y), \nabla_{X}^{* t} E\right\}$ belong to $\Gamma\left(S\left(T N^{\prime}\right)\right)$ and $\Gamma\left(\operatorname{Rad}\left(T N^{\prime}\right)\right)$, respectively.

By using above equations we obtain

$$
\begin{aligned}
& \breve{g}\left(h^{l}(X, J Y), E\right)=g\left(A_{E}^{*} X, J Y\right), \\
& \breve{g}\left(h^{*}(X, J Y), N\right)=g\left(A_{N} X, J Y\right), \\
& \breve{g}\left(h^{l}(X, E), E\right)=0, \quad A_{E}^{*} E=0 .
\end{aligned}
$$

Furthermore, Gauss equation is given by

$$
\begin{aligned}
\bar{R}(X, Y) Z= & R(X, Y) Z+A_{h^{l}(X, Z)} Y-A_{h^{l}(Y, Z)} X+A_{h^{s}(X, Z)} Y-A_{h^{s}(Y, Z)} X \\
& +\left(\nabla_{X} h^{l}\right)(Y, Z)-\left(\nabla_{Y} h^{l}\right)(X, Z)+D^{l}\left(X, h^{s}(Y, Z)\right)-D^{l}\left(Y, h^{s}(X, Z)\right) \\
& +\left(\nabla_{X} h^{s}\right)(Y, Z)-\left(\nabla_{Y} h^{s}\right)(X, Z)+D^{s}\left(X, h^{l}(Y, Z)\right)-D^{s}\left(Y, h^{l}(X, Z)\right) .
\end{aligned}
$$

for any $X, Y, Z \in \Gamma\left(T N^{\prime}\right)$.

\section{Semi-invariant lightlike submanifolds of semi-Riemannian golden manifolds}

Let $\left(N^{\prime}, g, S\left(T N^{\prime}\right), S\left(T N^{\prime}\right)\right)$ be a lightlike submanifold of a golden semi-Riemannian manifold $(\breve{N}, \breve{g}, \breve{P})$. Then, for any $X \in \Gamma\left(T N^{\prime}\right)$, we have

$$
\breve{P} X=P X+w X
$$

where $P X$ and $w X$ are the tangential and transversal components of $\breve{P} X$, respectively. Similarly, for any $U \in \operatorname{tr}\left(T N^{\prime}\right)$, we have

$$
\breve{P} U=B U+C U
$$

where $B U$ and $C U$ are the tangential and transversal components of $\breve{P} X$, respectively.

Definition 3.1. Let $N^{\prime}$ be a lightlike submanifold of a golden semi-Riemannian manifold $(\breve{N}, \breve{g}, \breve{P})$. If $\breve{P}\left(\operatorname{Rad}\left(T N^{\prime}\right)\right) \subset S\left(T N^{\prime}\right), \breve{P}\left(\operatorname{ltr}\left(T N^{\prime}\right)\right) \subset S\left(T N^{\prime}\right)$ and $\breve{P}\left(S\left(T N^{\prime \perp}\right)\right) \subset S\left(T N^{\prime}\right)$ then we call that $N^{\prime}$ is a semi-invariant lightlike submanifold.

If we set $D_{1}=\breve{P}\left(\operatorname{Rad}\left(T N^{\prime}\right)\right), D_{2}=\breve{P}\left(\operatorname{ltr}\left(T N^{\prime}\right)\right)$ and $D_{3}=\breve{P}\left(S\left(T N^{\prime \perp}\right)\right)$ then we have 
$S\left(T N^{\prime}\right)=D_{0} \perp\left\{D_{1} \oplus D_{2}\right\} \perp D_{3}$.

Thus we derive

$T N^{\prime}=D_{0} \perp\left\{D_{1} \oplus D_{2}\right\} \perp D_{3} \perp \operatorname{Rad}\left(T N^{\prime}\right)$,

$T \breve{N}=D_{0} \perp\left\{D_{1} \oplus D_{2}\right\} \perp D_{3} \perp S\left(T N^{\prime}\right) \perp\left\{\operatorname{Rad}\left(T N^{\prime}\right) \oplus \operatorname{ltr}\left(T N^{\prime}\right)\right\}$.

According to this definition we can write

$D=D_{0} \perp D_{1} \perp \operatorname{Rad}\left(T N^{\prime}\right)$

And

$D^{\perp}=D_{2} \perp D_{3}$.

Thus we have

$T N^{\prime}=D \oplus D^{\perp}$

For Case (2), we know that $S\left(T N^{\prime}\right)=\{0\}$. Then we derive

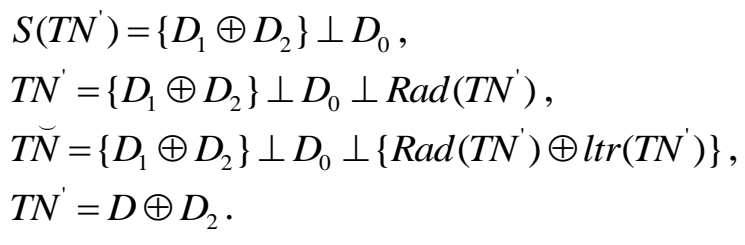

Proposition 3.2. The distribution $D_{0}$ are $D$ are invariant distributions with respect to $\breve{P}$.

Lemma 3.3. Let $N^{\prime}$ be a semi-invariant lightlike submanifold of a golden semiRiemannian manifold $(\breve{N}, \breve{g}, \breve{P})$ with $\bar{\nabla} \breve{P}=0$. Then, we have

$$
\begin{aligned}
& \left(\nabla_{X} P\right) Y=A_{w Y} X+B h(X, Y), \\
& w \nabla_{X} Y=\nabla_{X}^{t} w Y+h(X, P Y), \\
& \nabla_{X} B U=-P A_{U} X+B \nabla_{X}^{t} U, \\
& h(X, B U)=-w A_{U} X
\end{aligned}
$$

for any $X, Y \in \Gamma\left(T N^{\prime}\right)$ and $U \in \Gamma\left(\operatorname{tr}\left(T N^{\prime}\right)\right)$.

Throughout this paper, we assume $\bar{\nabla} \breve{P}=0$.

Lemma 3.4. Let $N^{\prime}$ be a semi-invariant lightlike submanifold of a golden semiRiemannian manifold $(\breve{N}, \breve{g}, \breve{P})$. Then, we have

$$
\begin{aligned}
& P^{2} X=P X+X-B w X, \\
& w P X=w X, P B U=B U, w B U=U,
\end{aligned}
$$


$g(P X, Y)-g(X, P Y)=g(X, w Y)-g(w X, Y)$,

$g(P X, P Y)=g(P X, Y)+g(X, Y)+g(w X, Y)-g(P X, w Y)-g(w X, P Y)-g(w X, w Y)$,

for any $X, Y \in \Gamma\left(T N^{\prime}\right)$ and $U \in \Gamma\left(\operatorname{tr}\left(T N^{\prime}\right)\right)$.

Lemma 3.5. Let $N^{\prime}$ be a lightlike submanifold of a golden semi-Riemannian manifold $(\breve{N}, \breve{g}, \breve{P})$. Then $P$ is golden structure on $N^{\prime}$ iff $w X=0$.

Example 3.6. Let $\left(\breve{N}=\mathbb{R}_{2}^{7}, \breve{g}\right)$ be a 7-dimensional semi-Euclidean space with signature $(-,-,+,+,+,+,+)$ and $\left(x_{1}, x_{2}, x_{3}, x_{4}, x_{5}, x_{6}, x_{7}\right)$ be the standard coordinate system of $\mathbb{R}_{2}^{7}$. If we define a mapping $\breve{P}$ by

$\breve{P}\left(x_{1}, x_{2}, x_{3}, x_{4}, x_{5}, x_{6}, x_{7}\right)=\left((1-\varphi) x_{1}, \varphi x_{2}, \varphi x_{3},(1-\varphi) x_{4}, \varphi x_{5},(1-\varphi) x_{6}, \varphi x_{7}\right)$ then $\breve{P}^{2}=\breve{P}+I$ and $\breve{P}$ is a golden structure on $\mathbb{R}_{2}^{7}$. Let $N^{\prime}$ be a submanifolds of $\breve{N}$ given by

$$
\begin{aligned}
& x_{1}=\varphi u_{1}+\frac{1}{2(2+\varphi)} u_{2}-u_{3}, \quad x_{2}=u_{1}-\frac{\varphi}{2(2+\varphi)} u_{2}+\varphi u_{3}, \\
& x_{3}=u_{1}+\frac{\varphi}{2(2+\varphi)} u_{2}+\varphi u_{3}, \quad x_{4}=\varphi u_{1}-\frac{1}{2(2+\varphi)} u_{2}-u_{3}, \\
& x_{5}=-u_{4}, \quad x_{6}=-u_{4}-u_{5}, \quad x_{7}=\varphi u_{4}+\varphi u_{5},
\end{aligned}
$$

where $u_{i}, 1 \leq i \leq 5$, are real parameters. Thus $T N^{\prime}=S p\left\{U_{1}, U_{2}, U_{3}, U_{4}, U_{5}\right\}$ where,

$$
\begin{aligned}
& U_{1}=\varphi \frac{\partial}{\partial x_{1}}+\frac{\partial}{\partial x_{2}}+\frac{\partial}{\partial x_{3}}+\varphi \frac{\partial}{\partial x_{4}}, U_{2}=\frac{1}{2(2+\varphi)}\left(\frac{\partial}{\partial x_{1}}-\varphi \frac{\partial}{\partial x_{2}}+\varphi \frac{\partial}{\partial x_{3}}-\frac{\partial}{\partial x_{4}}\right) \\
& U_{3}=-\frac{\partial}{\partial x_{1}}+\varphi \frac{\partial}{\partial x_{2}}+\varphi \frac{\partial}{\partial x_{3}}-\frac{\partial}{\partial x_{4}}, U_{4}=-\frac{\partial}{\partial x_{5}}-\frac{\partial}{\partial x_{6}}+\varphi \frac{\partial}{\partial x_{7}}, U_{5}=-\frac{\partial}{\partial x_{6}}+\varphi \frac{\partial}{\partial x_{7}}
\end{aligned}
$$

Then $N^{\prime}$ is a 1 -lightlike submanifold. We have $\operatorname{Rad}\left(T N^{\prime}\right)=\operatorname{Sp}\left\{E=U_{1}\right\}$ and $S\left(T N^{\prime}\right)=S p\left\{U_{2}, U_{3}, U_{4}, U_{5}\right\}$. Therefore, we derive

$$
\operatorname{ltr}\left(T N^{\prime}\right)=\operatorname{Sp}\left\{N=-\frac{1}{2(2+\varphi)}\left(\varphi \frac{\partial}{\partial x_{1}}+\frac{\partial}{\partial x_{2}}-\frac{\partial}{\partial x_{3}}-\varphi \frac{\partial}{\partial x_{4}}\right)\right\}
$$

And

$$
S\left(T N^{\prime \perp}\right)=S p\left\{L=\varphi \frac{\partial}{\partial x_{6}}+\frac{\partial}{\partial x_{7}}\right\}
$$

Furthermore, we get

$$
\breve{P} E=U_{3}, \breve{P} N=U_{2}, \breve{P} L=U_{4} .
$$


If we set $D_{0}=S p\left\{U_{5}\right\}, \quad D_{1}=\operatorname{Sp}\left\{U_{3}\right\}, \quad D_{2}=\operatorname{Sp}\left\{U_{2}\right\}, \quad D_{3}=\operatorname{Sp}\left\{U_{4}\right\}$, then $N^{\prime}$ is a semiinvariant lightlike submanifold of $\breve{N}$.

Theorem 3.7. Let $N^{\prime}$ be a semi-invariant lightlike submanifold of a golden semiRiemannian manifold $(\breve{N}, \breve{g}, \breve{P})$. Then $D$ is integrable iff

$h^{l}(\breve{P} X, \breve{P} Y)=h^{l}(\breve{P} X, Y)+h^{l}(X, Y), h^{s}(\breve{P} X, \breve{P} Y)=h^{s}(\breve{P} X, Y)+h^{s}(X, Y)$

for any $X, Y \in \Gamma(D)$.

Proof. For any $X, Y \in \Gamma(D), E \in \Gamma\left(\operatorname{Rad}\left(T N^{\prime}\right)\right), L \in \Gamma\left(S\left(T N^{\prime}\right)\right.$ and $N \in \Gamma\left(\operatorname{ltr}\left(T N^{\prime}\right)\right)$ the distribution $D$ is integrable iff

$\breve{g}([\breve{P} X, Y], \breve{P} E)=\breve{g}([\breve{P} X, Y], \breve{P} L)=0$.

Then, from (2.1) and (2.12) we obtain

$$
\begin{aligned}
& \breve{g}([\breve{P} X, Y], \breve{P} E)=\breve{g}\left(h^{l}(\breve{P} X, \breve{P} Y)-h^{l}(Y, \breve{P} X)-h^{l}(Y, X), E\right), \\
& \breve{g}([\breve{P} X, Y], \breve{P} L)=\breve{g}\left(h^{s}(\breve{P} X, \breve{P} Y)-h^{s}(Y, \breve{P} X)-h^{s}(Y, X), L\right)
\end{aligned}
$$

which completes the proof.

Theorem 3.8. Let $N^{\prime}$ be a semi-invariant lightlike submanifold of a golden semiRiemannian manifold $(\breve{N}, \breve{g}, \breve{P})$. If $D$ is integrable, then the leaves of $D$ have a golden structure.

Proof. Let $N^{\prime}$ be a semi-invariant lightlike submanifold and $M^{\prime}$ a leaf of $D$. Since $D$ is integrable, then for any $p \in M^{\prime}$, we have $T_{p} M^{\prime}=(D)_{p}$.

Letting $P^{\prime}=P_{\left.\right|_{D}}$, we say that $P^{\prime}$ defines an $(1,1)$-tensor field on $M^{\prime}$ because $D$ is $\breve{P}$ invariant.

If $X \in \Gamma(D)$, then $w X=0$. Thus we derive

$$
P^{\prime 2} X=P^{2} X=\breve{P}^{2} X=\breve{P} X+X=P X+X=P^{\prime} X+X
$$

which proves our assertion.

Theorem 3.9. Let $N^{\prime}$ be a semi-invariant lightlike submanifolds of a golden semiRiemannian manifold $(\breve{N}, \breve{g}, \breve{P})$. Then the distribution $D^{\perp}$ is integrable iff

(i) $\breve{g}\left(h^{*}(X, Y)-h^{*}(Y, X), N\right)=\breve{g}\left(A_{U_{2}} X-A_{U_{1}} Y, N\right)$,

(ii) $\breve{g}\left(A_{U_{1}} Y, \breve{P} N\right)=\breve{g}\left(A_{U_{2}} X, \breve{P} N\right)$,

(iii) $\breve{g}\left(A_{U_{1}} Y, \breve{P} Z\right)=\breve{g}\left(A_{U_{2}} X, \breve{P} Z\right)$, 
for any $X, Y \in \Gamma\left(D^{\perp}\right), Z \in \Gamma\left(D_{0}\right), \quad N \in \Gamma\left(\operatorname{ltr}\left(T N^{\prime}\right)\right)$ and $U_{1}, U_{2} \in \Gamma\left(\operatorname{tr}\left(T N^{\prime}\right)\right)$.

Proof. For any $X, Y \in \Gamma\left(D^{\perp}\right), Z \in \Gamma\left(D_{0}\right)$ and $N \in \Gamma\left(\operatorname{ltr}\left(T N^{\prime}\right)\right)$ the distribution $D^{\perp}$ is integrable iff

$\breve{g}([X, Y], \breve{P} N)=\breve{g}([X, Y], N)=\breve{g}([X, Y], Z)=0$

Choosing $X, Y \in \Gamma\left(D^{\perp}\right)$, there is a vector field $U_{1}, U_{2} \in \Gamma\left(\operatorname{tr}\left(T N^{\prime}\right)\right)$ such that $X=\breve{P} U_{1}$ and $Y=\breve{P} U_{2}$. Then, from (2.1), (2.11), (2.12) and (2.17) we derive

$$
\begin{aligned}
\breve{g}([X, Y], \breve{P} N) & =\breve{g}\left(\bar{\nabla}_{X} Y-\bar{\nabla}_{Y} X, \breve{P} N\right)=\breve{g}\left(\bar{\nabla}_{X} \breve{P} U_{2}-\bar{\nabla}_{Y} \breve{P} U_{1}, \breve{P} N\right) \\
& =\breve{g}\left(\bar{\nabla}_{X} \breve{P} U_{2}-\bar{\nabla}_{Y} \breve{P} U_{1}, N\right)+\breve{g}\left(\bar{\nabla}_{X} U_{2}-\bar{\nabla}_{Y} U_{1}, N\right) \\
& =\breve{g}\left(h^{*}\left(X, \breve{P} U_{2}\right)-h^{*}\left(Y, \breve{P} U_{1}\right), N\right)-\breve{g}\left(A_{U_{2}} X-A_{U_{1}} Y, N\right), \\
\breve{g}([X, Y], N)= & \breve{g}\left(\bar{\nabla}_{X} Y-\bar{\nabla}_{Y} X, N\right)=\breve{g}\left(\bar{\nabla}_{X} \breve{P} U_{2}-\bar{\nabla}_{Y} \breve{P} U_{1}, N\right) \\
& =\breve{g}\left(\bar{\nabla}_{X} U_{2}-\bar{\nabla}_{Y} U_{1}, \breve{P} N\right)=\breve{g}\left(A_{U_{1}} Y-A_{U_{2}} X, \breve{P} N\right), \\
\breve{g}([X, Y], Z)= & \breve{g}\left(\bar{\nabla}_{X} Y-\bar{\nabla}_{Y} X, Z\right)=\breve{g}\left(\bar{\nabla}_{X} \breve{P} U_{2}-\bar{\nabla}_{Y} \breve{P} U_{1}, Z\right) \\
= & \breve{g}\left(\bar{\nabla}_{X} U_{2}-\bar{\nabla}_{Y} U_{1}, \breve{P} Z\right)=\breve{g}\left(A_{U_{1}} Y-A_{U_{2}} X, \breve{P} Z\right) .
\end{aligned}
$$

This completes our proof.

Theorem 3.10. Let $N^{\prime}$ be a semi-invariant lightlike submanifold of a golden semiRiemannian manifold $(\breve{N}, \breve{g}, \breve{P})$. Then $\operatorname{Rad}\left(T N^{\prime}\right)$ is integrable iff

(i) $g\left(h^{*}\left(E, \breve{P} E^{\prime}\right), N\right)=\breve{g}\left(h^{*}\left(E^{\prime}, \breve{P} E\right), N\right)$,

(ii) $\breve{g}\left(h^{l}\left(E, \breve{P} E^{\prime}\right), E_{1}\right)=\breve{g}\left(h^{l}\left(E^{\prime}, \breve{P} E\right), E_{1}\right)$,

(iii) $\breve{g}\left(h^{s}\left(E, \breve{P} E^{\prime}\right), L\right)=\breve{g}\left(h^{s}(E, \breve{P} E), L\right)$,

(iv) $g\left(A_{E}^{*} E^{\prime}, X\right)=g\left(A_{E}^{*} E, X\right)$,

for any $X \in \Gamma\left(D_{0}\right), E, E^{\prime}, E_{1} \in \Gamma\left(\operatorname{Rad}\left(T N^{\prime}\right)\right), \quad N \in \Gamma\left(\operatorname{ltr}\left(T N^{\prime}\right)\right), L \in \Gamma\left(S\left(T N^{\prime \perp}\right)\right)$.

Proof. $\operatorname{Rad}\left(T N^{\prime}\right)$ is integrable iff

$$
\breve{g}\left(\left[E, E^{\prime}\right], \breve{P} N\right)=\breve{g}\left(\left[E, E^{\prime}\right], \breve{P} E_{1}\right)=\breve{g}\left(\left[E, E^{\prime}\right], \breve{P} L\right)=\breve{g}\left(\left[E, E^{\prime}\right], X\right)=0
$$

for any $X \in \Gamma\left(D_{0}\right), E, E^{\prime}, E_{1} \in \Gamma\left(\operatorname{Rad}\left(T N^{\prime}\right)\right), \quad N \in \Gamma\left(\operatorname{ltr}\left(T N^{\prime}\right)\right), L \in \Gamma\left(S\left(T N^{\prime \perp}\right)\right)$. Then, from (2.12), (2.17) and (2.18) we get

$$
\begin{aligned}
\breve{g}\left(\left[E, E^{\prime}\right], \breve{P} N\right) & =\breve{g}\left(\bar{\nabla}_{E} E^{\prime}-\bar{\nabla}_{E} E, \breve{P} N\right)=\breve{g}\left(\bar{\nabla}_{E} \breve{P} E^{\prime}-\bar{\nabla}_{E} \breve{P} E, N\right) \\
& =\breve{g}\left(h^{*}\left(E, \breve{P} E^{\prime}\right)-h^{*}\left(E^{\prime}, \breve{P} E\right), N\right), \\
\breve{g}\left(\left[E, E^{\prime}\right], \breve{P} E_{1}\right) & =\breve{g}\left(\bar{\nabla}_{E} E^{\prime}-\bar{\nabla}_{E^{\prime}} E, \breve{P} E_{1}\right)=\breve{g}\left(\bar{\nabla}_{E} \breve{P} E^{\prime}-\bar{\nabla}_{E} \breve{P} E, E_{1}\right) \\
& =\breve{g}\left(h^{l}\left(E, \breve{P} E^{\prime}\right)-h^{l}\left(E^{\prime}, \breve{P} E\right), E_{1}\right),
\end{aligned}
$$




$$
\begin{aligned}
\breve{g}\left(\left[E, E^{\prime}\right], \breve{P} L\right) & =\breve{g}\left(\bar{\nabla}_{E} E^{\prime}-\bar{\nabla}_{E} E, \breve{P} L\right)=\breve{g}\left(\bar{\nabla}_{E} \breve{P} E^{\prime}-\bar{\nabla}_{E} \breve{P} E, L\right) \\
& =\breve{g}\left(h^{s}\left(E, \breve{P} E^{\prime}\right)-h^{s}\left(E^{\prime}, \breve{P} E\right), L\right), \\
\breve{g}\left(\left[E, E^{\prime}\right], X\right) & =\breve{g}\left(\bar{\nabla}_{E} E^{\prime}-\bar{\nabla}_{E} E, X\right)=g\left(A_{E}^{*} E^{\prime}-A_{E}^{*} E, X\right)=0 .
\end{aligned}
$$

This completes the proof.

Theorem 3.11. Let $N^{\prime}$ be a semi-invariant lightlike submanifold of a golden semiRiemannian manifold $(\breve{N}, \breve{g}, \breve{P})$. Then, $\breve{P}\left(\operatorname{Rad}\left(T N^{\prime}\right)\right)$ is integrable iff

(i) $\breve{g}\left(h^{l}\left(\breve{P} E_{1}, E_{2}\right), E\right)=\breve{g}\left(h^{l}\left(\breve{P} E_{2}, E_{1}\right), E\right)$,

(ii) $\breve{g}\left(h^{s}\left(\breve{P} E_{1}, E_{2}\right), L\right)=\breve{g}\left(h^{s}\left(\breve{P} E_{2}, E_{1}\right), L\right)$

(iii) $g\left(A_{N} \breve{P} E_{1}, \breve{P} E_{2}\right)=g\left(A_{N} \breve{P} E_{2}, \breve{P} E_{1}\right)$,

(iv) $g\left(A_{E_{1}}^{*} \breve{P} E_{2}, \breve{P} X\right)=g\left(A_{E_{2}}^{*} \breve{P} E_{1}, \breve{P} X\right)$,

for any $X \in \Gamma\left(D_{0}\right), E, E^{\prime}, E_{1} \in \Gamma\left(\operatorname{Rad}\left(T N^{\prime}\right)\right), N \in \Gamma\left(\operatorname{ltr}\left(T N^{\prime}\right)\right), L \in \Gamma\left(S\left(T N^{\prime \perp}\right)\right)$.

Proof. $\breve{P} R a d\left(T N^{\prime}\right)$ is integrable iff

$$
\breve{g}\left(\left[\breve{P} E_{1}, \breve{P} E_{2}\right], \breve{P} E\right)=\breve{g}\left(\left[\breve{P} E_{1}, \breve{P} E_{2}\right], \breve{P} L\right)=\breve{g}\left(\left[\breve{P} E_{1}, \breve{P} E_{2}\right], N\right)=\breve{g}\left(\left[\breve{P} E_{1}, \breve{P} E_{2}\right], X\right)=0
$$

for any $X \in \Gamma\left(D_{0}\right), E, E^{\prime}, E_{1} \in \Gamma\left(\operatorname{Rad}\left(T N^{\prime}\right)\right), N \in \Gamma\left(\operatorname{ltr}\left(T N^{\prime}\right)\right), L \in \Gamma\left(S\left(T N^{\prime \perp}\right)\right)$. Since $\bar{\nabla}$ is a metric connection, from (2.1), (2.12), (2.13) and (2.18) we obtain

$$
\begin{aligned}
\breve{g}\left(\left[\breve{P} E_{1}, \breve{P} E_{2}\right], \breve{P} E\right)= & \breve{g}\left(\bar{\nabla}_{\breve{P} E_{1}} \breve{P} E_{2}-\bar{\nabla}_{\breve{P} E_{2}} \breve{P} E_{1}, \breve{P} E\right) \\
= & \breve{g}\left(\bar{\nabla}_{\breve{P} E_{1}} \breve{P} E_{2}, \breve{P} E\right)-\breve{g}\left(\bar{\nabla}_{\breve{P} E_{2}} \breve{P} E_{1}, \breve{P} E\right) \\
= & \breve{g}\left(\bar{\nabla}_{\breve{P} E_{1}} \breve{P} E_{2}, E\right)+\breve{g}\left(\bar{\nabla}_{\breve{P} E_{1}} E_{2}, E\right)-\breve{g}\left(\bar{\nabla}_{\breve{P} E_{2}} \breve{P} E_{1}, E\right)-\breve{g}\left(\bar{\nabla}_{\breve{P} E_{2}} E_{1}, E\right) \\
= & \breve{g}\left(h^{l}\left(\breve{P} E_{1}, \breve{P} E_{2}\right), E\right)+\breve{g}\left(h^{l}\left(\breve{P} E_{1}, E_{2}\right), E\right) \\
& -\breve{g}\left(h^{l}\left(\breve{P} E_{2}, \breve{P} E_{1}\right), E\right)-\breve{g}\left(h^{l}\left(\breve{P} E_{2}, E_{1}\right), E\right) \\
= & \breve{g}\left(h^{l}\left(\breve{P} E_{1}, E_{2}\right), E\right)-\breve{g}\left(h^{l}\left(\breve{P} E_{2}, E_{1}\right), E\right), \\
\breve{g}\left(\left[\breve{P} E_{1}, \breve{P} E_{2}\right], \breve{P} L\right)= & \breve{g}\left(\bar{\nabla}_{\breve{P} E_{1}} \breve{P} E_{2}-\bar{\nabla}_{\breve{P} E_{2}} \breve{P} E_{1}, \breve{P} L\right) \\
= & \breve{g}\left(\bar{\nabla}_{\breve{P} E_{1}} \breve{P} E_{2}, \breve{P} L\right)-\breve{g}\left(\bar{\nabla}_{\breve{P} E_{2}} \breve{P} E_{1}, \breve{P} L\right) \\
= & \breve{g}\left(\bar{\nabla}_{\breve{P} E_{1}} \breve{P} E_{2}, L\right)+\breve{g}\left(\bar{\nabla}_{\breve{P}_{1}} E_{2}, L\right)-\breve{g}\left(\bar{\nabla}_{\breve{P} E_{2}} \breve{P} E_{1}, L\right)-\breve{g}\left(\bar{\nabla}_{\breve{P} E_{2}} E_{1}, L\right) \\
= & \breve{g}\left(h^{s}\left(\breve{P} E_{1}, \breve{P} E_{2}\right), L\right)+\breve{g}\left(h^{s}\left(\breve{P} E_{1}, E_{2}\right), L\right) \\
& -\breve{g}\left(h^{s}\left(\breve{P} E_{2}, \breve{P} E_{1}\right), L\right)-\breve{g}\left(h^{s}\left(\breve{P} E_{2}, E_{1}\right), L\right) \\
= & \breve{g}\left(h^{s}\left(\breve{P} E_{1}, E_{2}\right), L\right)-\breve{g}\left(h^{s}\left(\breve{P} E_{2}, E_{1}\right), L\right), \\
\breve{g}\left(\left[\breve{P} E_{1}, \breve{P} E_{2}\right], N\right)= & \breve{g}\left(\bar{\nabla}_{\breve{P} E_{1}} \breve{P} E_{2}-\bar{\nabla}_{\breve{P} E_{2}} \breve{P} E_{1}, N\right)=\breve{g}\left(\bar{\nabla}_{\breve{P} E_{1}} \breve{P} E_{2}, N\right)-\breve{g}\left(\bar{\nabla}_{\breve{P} E_{2}} \breve{P} E_{1}, N\right) \\
= & -\breve{g}\left(\breve{P} E_{2}, \bar{\nabla}_{\breve{P} E_{1}} N\right)+\breve{g}\left(\breve{P} E_{1}, \bar{\nabla}_{\breve{P} E_{2}} N\right) \\
= & g\left(A_{N} \breve{P} E_{1}, \breve{P} E_{2}\right)-g\left(A_{N} P E_{2}, \breve{P} E_{1}\right),
\end{aligned}
$$




$$
\begin{aligned}
\breve{g}\left(\left[\breve{P} E_{1}, \breve{P} E_{2}\right], X\right) & =\breve{g}\left(\bar{\nabla}_{\bar{P} E_{1}} \breve{P} E_{2}-\bar{\nabla}_{\bar{P}_{2}} \breve{P} E_{1}, X\right)=\breve{g}\left(\bar{\nabla}_{\breve{P}_{1}} E_{2}, \breve{P} X\right)-\breve{g}\left(\bar{\nabla}_{\bar{P}_{2}} E_{1}, \breve{P} X\right) \\
& =g\left(A_{E_{1}}^{*} \breve{P} E_{2}-A_{E_{2}}^{*} \breve{P} E_{1}, \breve{P} X\right)=0 .
\end{aligned}
$$

Thus the proof is completed.

Theorem 3.12. Let $N^{\prime}$ be a semi invariant lightlike submanifold of a golden semiRiemannian manifold $(\breve{N}, \breve{g}, \breve{P})$. Then, each leaf of radical distribution is totally geodesic on $N^{\prime}$ iff

(i) $A_{E_{2}}^{*} E_{1} \in \Gamma\left(D_{1} \perp D_{3}\right)$,

(ii) $\breve{g}\left(h^{*}\left(E_{1}, \breve{P} E_{2}\right), N\right)=0$,

(iii) $\breve{g}\left(h^{s}\left(E_{1}, \breve{P} E_{2}\right), L\right)=0$,

for any $E, E_{1}, E_{2} \in \Gamma\left(\operatorname{Rad}\left(T N^{\prime}\right)\right), N \in \Gamma\left(\operatorname{ltr}\left(T N^{\prime}\right)\right), L \in \Gamma\left(S\left(T N^{\prime \perp}\right)\right)$.

Proof. Radical distribution is totally geodesic iff

$$
g\left(\nabla_{E_{1}} E_{2}, \breve{P} E\right)=g\left(\nabla_{E_{1}} E_{2}, X\right)=g\left(\nabla_{E_{1}} E_{2}, \breve{P} N\right)=g\left(\nabla_{E_{1}} E_{2}, \breve{P} L\right)=0,
$$

for any $X \in \Gamma\left(D_{0}\right), \quad E, E_{1}, E_{2} \in \Gamma\left(\operatorname{Rad}\left(T N^{\prime}\right)\right), \quad N \in \Gamma\left(\operatorname{ltr}\left(T N^{\prime}\right)\right), \quad L \in \Gamma\left(S\left(T N^{\prime}\right)\right)$. Using (2.12), (2.17) and (2.18), we have

$$
\begin{aligned}
& g\left(\nabla_{E_{1}} E_{2}, \breve{P} E\right)=\breve{g}\left(\bar{\nabla}_{E_{1}} E_{2}, \breve{P} E\right)=-g\left(A_{E_{2}}^{*} E_{1}, \breve{P} E\right), \\
& g\left(\nabla_{E_{1}} E_{2}, X\right)=\breve{g}\left(\bar{\nabla}_{E_{1}} E_{2}, X\right)=-g\left(A_{E_{2}}^{*} E_{1}, X\right), \\
& \breve{g}\left(\nabla_{E_{1}} E_{2}, \breve{P} N\right)=\breve{g}\left(\bar{\nabla}_{E_{1}} E_{2}, \breve{P} N\right)=\breve{g}\left(h^{*}\left(E_{1}, \breve{P} E_{2}\right), N\right), \\
& \breve{g}\left(\nabla_{E_{1}} E_{2}, \breve{P} L\right)=\breve{g}\left(\bar{\nabla}_{E_{1}} E_{2}, \breve{P} L\right)=\breve{g}\left(h^{s}\left(E_{1}, \breve{P} E_{2}\right), L\right) .
\end{aligned}
$$

Hence, from (3.35)-(3.38) we complete the proof.

Definition 3.13. Let $N^{\prime}$ be a proper semi-invariant r-lightlike submanifold of a golden semi-Riemannian manifold $\vec{N}$. If

$h(X, Y)=0, \quad \forall X \in \Gamma(D)$ and $Y \in \Gamma\left(D^{\perp}\right)$

then, $N^{\prime}$ is called as mixed-geodesic submanifold.

Theorem 3.14. Let $N^{\prime}$ be a semi invariant lightlike submanifold of a golden semiRiemannian manifold $(\breve{N}, \breve{g}, \breve{P})$. Then the followings are equivalent:

i) $N^{\prime}$ is mixed geodesic,

ii) $A_{U} X$ has only component in $\Gamma(D)$,

iii) $A_{E}^{*} X$ and $A_{L} X$ have no components in $D_{1}$ and $D_{3}$.

iv) $\nabla_{Y}^{*} \breve{P} E, \nabla_{Y}^{*} \breve{P} L \in \Gamma\left(D_{0} \perp D_{2}\right)$

for any $X \in \Gamma(D), Y \in \Gamma\left(D^{\perp}\right), E \in \Gamma\left(\operatorname{Rad}\left(T N^{\prime}\right)\right), U \in \Gamma\left(\operatorname{tr}\left(T N^{\prime}\right)\right), L \in \Gamma\left(S\left(T N^{\prime \perp}\right)\right)$. 
Proof. $N^{\prime}$ is mixed-geodesic iff

$\breve{g}(h(X, Y), E)=0$ and $\breve{g}(h(X, Y), L)=0$

for any $X \in \Gamma(D), Y \in \Gamma\left(D^{\perp}\right), E \in \Gamma\left(\operatorname{Rad}\left(T N^{\prime}\right)\right), L \in \Gamma\left(S\left(T N^{\prime}\right)\right)$. Choosing $Y \in \Gamma\left(D^{\perp}\right)$, there is a vector field $U \in \Gamma(\operatorname{tr}(T M))$ such that $Y=\breve{P} U$. Using (2.10) and (2.11) we have

$$
\begin{aligned}
\breve{g}(h(X, Y), E) & =\breve{g}\left(\bar{\nabla}_{X} Y, E\right)=\breve{g}\left(\bar{\nabla}_{X} \breve{P} U, E\right) \\
& =\breve{g}\left(\bar{\nabla}_{X} U, \breve{P} E\right)=-\breve{g}\left(A_{U} X, \breve{P} E\right), \\
\breve{g}(h(X, Y), L) & =\breve{g}\left(\bar{\nabla}_{X} Y, L\right)=\breve{g}\left(\bar{\nabla}_{X} \breve{P} U, L\right) \\
& =\breve{g}\left(\bar{\nabla}_{X} U, \breve{P} L\right)=-\breve{g}\left(A_{U} X, \breve{P} L\right) .
\end{aligned}
$$

Thus we derive $(i) \Leftrightarrow(i i)$. From (2.10), (2.14) and (2.18) we get

$$
\begin{aligned}
& \breve{g}(h(X, Y), E)=g\left(Y, A_{E}^{*} X\right), \\
& \breve{g}(h(X, Y), L)=\breve{g}\left(Y, A_{L} X-D^{l}(X, L)\right) .
\end{aligned}
$$

Hence we obtain $(i) \Leftrightarrow($ iii $)$. Since $\bar{\nabla}$ is a metric connection, from (2.10) we derive

$$
\begin{aligned}
\breve{g}(h(\breve{P} X, Y), E) & =\breve{g}(h(Y, \breve{P} X), E)=\breve{g}\left(\bar{\nabla}_{Y} \breve{P} X, E\right)=-\breve{g}\left(\breve{P} X, \bar{\nabla}_{Y} E\right) \\
& =-\breve{g}\left(X, \bar{\nabla}_{Y} \breve{P} E\right)=-g\left(X, \nabla_{Y}^{*} \breve{P} E\right), \\
\breve{g}(h(\breve{P} X, Y), L) & =\breve{g}(h(Y, \breve{P} X), L)=\breve{g}\left(\bar{\nabla}_{Y} \breve{P} X, L\right)=-\breve{g}\left(\breve{P} X, \bar{\nabla}_{Y} L\right) \\
& =-\breve{g}\left(X, \bar{\nabla}_{Y} \breve{P} L\right)=-g\left(X, \nabla_{Y}^{*} \breve{P} L\right) .
\end{aligned}
$$

Thus we derive $(i) \Leftrightarrow(i v)$.

Definition 3.15. A semi-invariant submanifold $N^{\prime}$ of a golden semi-Riemannian manifold $N^{\prime}$ is named as $D$-totally geodesic (resp. $D^{\perp}$-totally geodesic) if its the second fundamental form $h$ satisfies $h(X, Y)=0$ (resp. $h(Z, L)=0$ ), for any $X, Y \in \Gamma(D)$, $\left(Z, L \in \Gamma\left(D^{\perp}\right)\right)$.

Theorem 3.16. Let $N^{\prime}$ be a semi-invariant lightlike submanifold of a golden semiRiemannian manifold $(\breve{N}, \breve{g}, \breve{P})$. Then the followings are equivalent:

(i) $N^{\prime}$ is $D$-geodesic,

(ii) $A_{E}^{*} X \in \Gamma\left(D_{1} \perp D_{3}\right)$ and $g\left(Y, A_{L} X\right)=g\left(Y, D^{l}(X, L)\right)$,

(iii) $\nabla^{*}{ }_{X} Y$ has no components in $D_{2}$ and $D_{3}$,

(iv) $\nabla_{X}^{*} \breve{P} E, \nabla_{X}^{*} \breve{P} L \in \Gamma\left(D_{1} \perp D_{3}\right)$,

for any $X, Y \in \Gamma(D), E \in \Gamma\left(\operatorname{Rad}\left(T N^{\prime}\right)\right), L \in \Gamma\left(S\left(T N^{\prime \perp}\right)\right)$.

Proof. For any $X, Y \in \Gamma(D), E \in \Gamma\left(\operatorname{Rad}\left(T N^{\prime}\right)\right)$ and $L \in \Gamma\left(S\left(T N^{\prime \perp}\right)\right)$, using (2.10), (2.14) and (2.18) we obtain 
$\breve{g}(h(X, Y), E)=g\left(Y, A_{E}^{*} X\right)$,

And

$\breve{g}(h(X, Y), L)=\breve{g}\left(Y, A_{L} X-D^{l}(X, L)\right)$.

Hence we derive $(i) \Rightarrow(i i)$. Since $\bar{\nabla}$ is a metric connection, from (2.10), (2.14) and (2.18) we get

$$
\begin{aligned}
g\left(\nabla_{X}^{*} Y, \breve{P} E\right) & =\breve{g}\left(\bar{\nabla}_{X} Y, \breve{P} E\right)=\breve{g}\left(\bar{\nabla}_{X} \breve{P} Y, E\right) \\
& =-\breve{g}\left(\breve{P} Y, \bar{\nabla}_{X} E\right)=\breve{g}\left(\breve{P} Y, A_{E}^{*} X\right) . \\
g\left(\nabla_{X}^{*} Y, \breve{P} L\right) & =\breve{g}\left(\bar{\nabla}_{X} Y, \breve{P} L\right)=\breve{g}\left(\bar{\nabla}_{X} \breve{P} Y, L\right) \\
& =-\breve{g}\left(\breve{P} Y, \bar{\nabla}_{X} L\right)=\breve{g}\left(\breve{P} Y, A_{L} X-D^{l}(X, L)\right) .
\end{aligned}
$$

This is (ii) $\Rightarrow$ (iii). Similarly, since $\bar{\nabla}$ is a metric connection, from (2.10) and (2.17) we have

$$
g\left(\nabla_{X}^{*} \breve{P} E, Y\right)=\breve{g}\left(\bar{\nabla}_{X} \breve{P} E, Y\right)=-\breve{g}\left(\breve{P} E, \bar{\nabla}_{X} Y\right)=-\breve{g}\left(\breve{P} E, \nabla_{X}^{*} Y\right)
$$

And

$$
g\left(\nabla_{X}^{*} \breve{P} L, Y\right)=\breve{g}\left(\bar{\nabla}_{X} \breve{P} L, Y\right)=-\breve{g}\left(\breve{P} L, \bar{\nabla}_{X} Y\right)=-\breve{g}\left(\breve{P} L, \nabla_{X}^{*} Y\right)
$$

Hence we get $(i i i) \Rightarrow(i v)$. Since $\bar{\nabla}$ is a metric connection, using (2.1), (2.10) and (2.17) we derive

$$
\begin{aligned}
\breve{g}(h(X, Y), E) & =\breve{g}\left(\bar{\nabla}_{X} Y, E\right)=\breve{g}\left(\bar{\nabla}_{X} \breve{P} Y, \breve{P} E\right)-\breve{g}\left(\bar{\nabla}_{X} Y, \breve{P} E\right) \\
& =-\breve{g}\left(\breve{P} Y, \bar{\nabla}_{X} \breve{P} E\right)+\breve{g}\left(Y, \bar{\nabla}_{X} \breve{P} E\right)=-\breve{g}\left(\breve{P} Y, \nabla_{X}^{*} \breve{P} E\right)+\breve{g}\left(Y, \nabla_{X}^{*} \breve{P} E\right) . \\
\breve{g}(h(X, Y), L) & =\breve{g}\left(\bar{\nabla}_{X} Y, L\right)=\breve{g}\left(\bar{\nabla}_{X} \breve{P} Y, \breve{P} L\right)-\breve{g}\left(\bar{\nabla}_{X} Y, \breve{P} L\right) \\
& =-\breve{g}\left(\breve{P} Y, \bar{\nabla}_{X} \breve{P} L\right)+\breve{g}\left(Y, \bar{\nabla}_{X} \breve{P} L\right)=-\breve{g}\left(\breve{P} Y, \nabla_{X}^{*} \breve{P} L\right)+\breve{g}\left(Y, \nabla_{X}^{*} \breve{P} L\right) .
\end{aligned}
$$

Thus we get $(i v) \Rightarrow(i)$.

Theorem 3.17. Let $N^{\prime}$ be a semi-invariant lightlike submanifold of a golden semiRiemannian $(\breve{N}, \breve{g}, \breve{P})$. Then the following statements are equivalent:

(i) $N^{\prime}$ is $D^{\perp}$-geodesic,

(ii) $A_{E}^{*} X$ and $A_{L} X$ have no components in $D_{1}$ and $D_{3}$,

(iii) $A_{U} X$ has no components in $D_{2}$ and $D_{3}$,

for any $X, Y \in \Gamma\left(D^{\perp}\right), E \in \Gamma\left(\operatorname{Rad}\left(T N^{\prime}\right)\right), U \in \Gamma\left(\operatorname{tr}\left(T N^{\prime}\right)\right), L \in \Gamma\left(S\left(T N^{\prime \perp}\right)\right)$.

Proof. $N^{\prime}$ is $D^{\perp}-$ geodesic iff

$\breve{g}(h(X, Y), E)=0$ and $\breve{g}(h(X, Y), L)=0$, 
for any $X, Y \in \Gamma\left(D^{\perp}\right), E \in \Gamma\left(\operatorname{Rad}\left(T N^{\prime}\right)\right), L \in \Gamma\left(S\left(T N^{\prime \perp}\right)\right)$. From (2.10), (2.14) and (2.18) we get

$\breve{g}(h(X, Y), E)=g\left(Y, A_{E}^{*} X\right)$,

and

$\breve{g}(h(X, Y), L)=g\left(Y, A_{L} X-D^{l}(X, L)\right)$.

Thus we derive $(i) \Leftrightarrow(i i)$. Choosing $Y \in \Gamma\left(D^{\perp}\right)$, there is a vector field $U \in \Gamma\left(\operatorname{tr}\left(T N^{\prime}\right)\right)$ such that $Y=\breve{P} U$. Similarly from (2.10) and (2.11) we get

$$
\begin{aligned}
\breve{g}(h(X, Y), E) & =\breve{g}\left(\bar{\nabla}_{X} Y, E\right)=\breve{g}\left(\bar{\nabla}_{X} \breve{P} U, E\right) \\
& =\breve{g}\left(\bar{\nabla}_{X} U, \breve{P} E\right)=-\breve{g}\left(A_{U} X, \breve{P} E\right), \\
\breve{g}(h(X, Y), L) & =\breve{g}\left(\bar{\nabla}_{X} Y, L\right)=\breve{g}\left(\bar{\nabla}_{X} \breve{P} U, L\right) \\
& =\breve{g}\left(\bar{\nabla}_{X} U, \breve{P} L\right)=-\breve{g}\left(A_{U} X, \breve{P} L\right) .
\end{aligned}
$$

This is $(i) \Leftrightarrow(i i i)$.

Theorem 3.18. Let $N^{\prime}$ be a semi-invariant lightlike submanifold of a golden semiRiemannian manifold $(\breve{N}, \breve{g}, \breve{P})$. Then, following statements are equivalent.

(i) $D$ is parallel,

(ii) $N^{\prime}$ is $D$-geodesic,

(iii) $P$ is parallel on $N^{\prime}$.

Proof. Using (3.4) we have, $D$ is parallel iff $g\left(\nabla_{X} Y, \breve{P} E\right)=g\left(\nabla_{X} Y, \breve{P} L\right)=0$, for any $X, Y \in \Gamma(D), E \in \Gamma\left(\operatorname{Rad}\left(T N^{\prime}\right)\right), L \in \Gamma\left(S\left(T N^{\perp}\right)\right)$. From (2.12) we derive

$$
\begin{aligned}
& g\left(\nabla_{X} Y, \breve{P} E\right)=\breve{g}\left(h^{l}(X, \breve{P} Y), E\right), \\
& \breve{g}\left(\nabla_{X} Y, \breve{P} L\right)=\breve{g}\left(h^{s}(X, \breve{P} Y), L\right) .
\end{aligned}
$$

Hence we derive (i) $\Leftrightarrow$ (ii). From (3.13) for any $X, Y \in \Gamma(D)$ we have $\left(\nabla_{X} P\right) Y=B h(X, Y)$. Since $N^{\prime}$ is $D-$ geodesic, $\left(\nabla_{X} P\right) Y=0$. Thus we get (ii) $\Leftrightarrow$ (iii).

Now, by using the equation (2.4) and (3.1), we derive

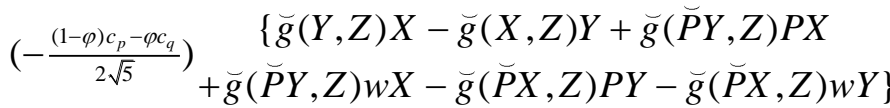

$$
\begin{aligned}
& \bar{R}(X, Y) Z=+\left(-\frac{(1-\varphi) c_{p}+\varphi c_{q}}{4}\right)\{\breve{g}(\breve{P} Y, Z) X+\breve{g}(Y, Z) P X+\breve{g}(Y, Z) w X \\
& -\breve{g}(\breve{P} X, Z) Y-\breve{g}(X, Z) P Y-\breve{g}(X, Z) w Y\}
\end{aligned}
$$

for any $X, Y, Z \in \Gamma\left(T N^{\prime}\right)$. From (2.22) and (3.57), the equations of Gauss and Codazzi for the submanifold $N^{\prime}$, respectively, can be written as 


$$
\left(-\frac{(1-\varphi) c_{p}-\varphi c_{q}}{2 \sqrt{5}}\right)\{\breve{g}(Y, Z) X-\breve{g}(X, Z) Y+\breve{g}(\breve{P} Y, Z) P X-\breve{g}(\breve{P} X, Z) P Y\}
$$

$$
\begin{gathered}
R(X, Y) Z=\quad+\left(-\frac{(1-\varphi) c_{p}+\varphi c_{q}}{4}\right)\{\breve{g}(\breve{P} Y, Z) X-\breve{g}(\breve{P} X, Z) Y \\
+\breve{g}(Y, Z) P X-\breve{g}(X, Z) P Y\} \\
+A_{h(Y, Z)} X-A_{h(X, Z)} Y .
\end{gathered}
$$

and

$$
\begin{aligned}
\left(\nabla_{X} h\right)(Y, Z)-\left(\nabla_{Y} h\right)(X, Z)= & \left(-\frac{(1-\varphi) c_{p}-\varphi c_{q}}{2 \sqrt{5}}\right)\{\breve{g}(\breve{P} Y, Z) w X-\breve{g}(\breve{P} X, Z) w Y\} \\
& +\left(-\frac{(1-\varphi) c_{p}+\varphi c_{q}}{4}\right)\{\breve{g}(Y, Z) w X-\breve{g}(X, Z) w Y\} .
\end{aligned}
$$

for any $X, Y, Z \in \Gamma\left(T N^{\prime}\right)$.

Definition 3.19. Let $N^{\prime}$ be an r-lightlike submanifold of any semi-Riemannian manifold $\breve{N} . N^{\prime}$ is called as curvature-invariant lightlike submanifold if for $\forall X, Y, Z \in \Gamma\left(T N^{\prime}\right)$,

$$
\left(\nabla_{X} h\right)(Y, Z)-\left(\nabla_{Y} h\right)(X, Z)=0 .
$$

Theorem 3.20. There is no curvature-invariant semi-invariant lightlike submanifolds in any semi-Riemannian locally golden product space form $\left(\breve{N}=N_{p}^{\prime}\left(c_{p}\right) \times N_{q}^{\prime}\left(c_{q}\right), \breve{g}, \breve{P}\right)$ with $c_{p}, c_{q} \neq 0$.

Proof. Suppose that $N^{\prime}$ is a semi curvature-invariant lightlike submanifold of a semiRiemannian golden product space form $\left(\breve{N}=N_{p}^{\prime}\left(c_{p}\right) \times N_{q}^{\prime}\left(c_{q}\right), \breve{g}, \breve{P}\right)$ with $c_{p}, c_{q} \neq 0$. Then from (3.59) we have

$$
\begin{gathered}
\left(-\frac{(1-\varphi) c_{p}-\varphi c_{q}}{2 \sqrt{5}}\right)\{\breve{g}(\breve{P} Y, Z) w X-\breve{g}(\breve{P} X, Z) w Y\} \\
+\left(-\frac{(1-\varphi) c_{p}+\varphi c_{q}}{4}\right)\{\breve{g}(Y, Z) w X-\breve{g}(X, Z) w Y\}=0 .
\end{gathered}
$$

If we take $X \in \Gamma\left(D_{3}\right), Y \in \Gamma\left(\operatorname{Rad}\left(T N^{\prime}\right)\right)$ and $Z \in \Gamma\left(D_{2}\right)$ in (3.61), then we get

$$
\left(-\frac{(1-\varphi) c_{p}-\varphi c_{q}}{2 \sqrt{5}}\right) \omega X=0
$$

Similarly, if we take $X \in \Gamma\left(D_{3}\right), Y \in \Gamma\left(D_{1}\right)$ and $Z \in \Gamma\left(D_{2}\right)$ in (3.61), then we get

$$
\left(-\frac{(1-\varphi) c_{p}-\varphi c_{q}}{2 \sqrt{5}}\right) \omega X+\left(-\frac{(1-\varphi) c_{p}+\varphi c_{q}}{4}\right) \omega Y=0 .
$$

Using the equations (3.62) and (3.63), we get $c_{p}, c_{q}=0$. 


\section{References}

[1] Acet, B. E., Lightlike hypersurfaces of metallic semi-Riemannian manifolds, Int. Jour. of Geometric method in modern Physics, 15(12), 185-201, (2018).

[2] Acet, B. E., Perktaş, S. Y. and Kılıç, E., Lightlike Submanifolds of a ParaSasakian Manifold, Gen. Math. Notes, 22(2), 22-45, (2014).

[3] Atçeken, M. and Kılıç, E., Semi-Invariant Lightlike Submanifolds of a SemiRiemannian Product Manifold, Kodai Math. J., 30(3), 361-378, (2007).

[4] Bahadır, O. and Kılıç, E., Lightlike submanifolds of indefinite Kaehler manifolds with quarter symmetric non-metric connection, Mathematical Sciences And Applications E-Notes, 2(2), 89-104, (2014).

[5] Bahadır, O. and Kılıç, E., Lightlike Submanifolds of a Semi-Riemannian Product Manifold with Quarter Symmetric Non-Metric Connection, International Electronic Journal of Geometry, 9(1), 9-22, (2016).

[6] Crasmareanu, M. and Hretcanu, C. E., Golden Differential Geometry, Chaos, Solitons \& Fractals, 38, 1229-1238, (2008).

[7] Duggal, K. L. and Bejancu, A., Lightlike submanifold of semi-Riemannian manifolds and its applications, Kluwer Academic, The Netherlands, (1996).

[8] Duggal, K. L. and Şahin, B., Screen Cauchy Riemann lightlike submanifolds, Acta Math. Hungar., 106(1-2), 137-165, (2005).

[9] Duggal, K. L. and Şahin, B., Generalized Cauchy Riemann lightlike submanifolds, Acta Math. Hungar., 112(1-2), 113-136, (2006).

[10] Duggal, K. L. and Şahin, B., Differential geometry of lightlike submanifolds, Birkhäuser Verlag, (2010).

[11] Erdoğan, F. E., Transversal lightlike submanifolds of metallic semi-Riemannian manifolds, Turkish Journal of Mathematics, 42 (6), 3133-3148, (2018).

[12] Erdoğan, F. E. and Y1ldırım, C., On a study of the totally umbilical semiinvariant submanifolds of golden Riemannian manifolds, Politeknik Dergisi 21 (4), 967-970, (2018).

[13] Gezer, A., Cengiz, N., Salimov, A., On integrability of golden Riemannian structures, Turk. J. Math., 37, 693-703, (2013).

[14] Goldberg S.I., Yano K., Polynomial structures on manifolds, Kodai Math. Sem. Rep., 22, 199-218, (1970).

[15] Hretcanu, C. E., Crasmareanu, M., On some invariant submanifolds in Riemannian manifold with golden structure, An. Ştiint. Univ. Al. I. Cuza Iaşi. Mat. (N.S.), 53, suppl. 1, 199-211, (2007).

[16] Kılıç, E. and Şahin, B., Radical anti-invariant lightlike submanifolds of a SemiRiemannian Product Manifold, Turkish Journal of Mathematics, 32, 429-449, (2008).

[17] Kupeli, D. N., Singular Semi-Riemannian Geometry, Kluwer Academic Publishers, Dordrecht, (1996).

[18] Özkan, M., Prolongations of golden structures to tangent bundles, Diff. Geom. Dyn. Syst., 16, 227-238, (2014).

[19] (Önen) Poyraz, N. and Yaşar, E., Lightlike hypersurfaces of a golden semiRiemannian manifold, Mediterr. J. Math., 14:204, (2017).

[20] Yano, K., On a structure defined by a tensor field $f$ of type $(1,1)$ satisfying $f^{3}+f=0$, Tensor, N.S., 14, 99-109, (1963).

[21] Yano, K. and Kon, M., Structure on manifolds, World Scientific Publishing, (1984). 